\title{
The effect of "significance" upon indices of Sokolov's orienting response: A new conceptualization to replace the OR
}

\author{
ROBERT J. BARRY \\ School of Education, University of New South Wales, Kensington 2033, Australia
}

\begin{abstract}
Sokolov's conceptualization of the OR has many elaborations reflecting its postulated importance in perceptual and motor processes, but in the final analysis its validity rests on the reality of the physiological processes which are hypothesized to occur in response to simple stimuli. These are supposed to behave in a coherent manner which allows the use of the term "OR" as a unitary concept. Such an OR is hypothesized to depend on stimulus intensity, novelty, and significance in well-defined ways. This study focused upon the last variable as manipulated by attentional demand. The small effects obtained by this manipulation were not as predicted by Sokolov, and were able to be explained most parsimoniously in terms of novelty. These results generated a new hypothetical structure tentatively offered as a first step in the replacement of the unitary OR concept.
\end{abstract}

Sokolov's concept of the orienting response (OR) can be most simply described as a unitary nonspecific response elicited by novel stimuli. The dependence of the response complex upon stimulus novelty is reflected in what has become recognized as an essential descriptor of the OR, its habituation with stimulus repetition (Graham, 1973). Another important determinant of the OR is the intensity of the eliciting stimulus, since within the moderate range of stimulus intensity, there is claimed to be "direct dependence on the intensity of the stimulus" (Sokolov, 1963, p. 12). Most of the published work in the OR field has been relevant to the influence of variation in these two independent variables upon OR magnitude. This has used what might be termed "indifferent" stimuli in the sense that the stimulus has no significance for the organism apart from its physical parameters. Sokolov also claims that the subject may attribute additional significance to a stimulus due to prior conditioning or the experimenter's instructions, which modify the perception of that stimulus so that it becomes a "signal stimulus": "In its broad meaning the term 'signal stimulus' is applied to those stimuli which evoke a reaction in anticipation of external agents likely to appear in the future .... In the case of man the term ... should also be applied to stimuli to which activity is directed by implication"' (Sokolov, 1963, p. 163).

It would thus appear that instructions to attend or respond to the stimulus array modify the response generated by the stimulus: "With signal stimuli, extinction of the orientation reflex is much slower than with non-signal stimuli. For instance, whereas

Address requests for reprints to Robert J. Barry, School of Education, University of N.S.W., Kensington 2033, Australia. the orientation reaction to a non-signal agent becomes extinguished after 3-12 applications, a signal stimulus can evoke it for dozens of times" (Sokolov, 1963 , p. 164). In addition to this change in habituation rate, a signal stimulus apparently also produces an enhanced OR magnitude, since Sokolov has written of: "the increased intensity of the reaction as a result of verbal instructions" (Sokolov, 1963, p. 189). Thus specific predictions can be generated concerning the magnitude of the OR as a function of stimulus intensity and novelty when the eliciting conditions are varied along the dimension of significance from "indifferent" to "signal." Such a variation can supposedly be controlled by the experimenter's instructions to attend or respond to the stimulus complex in various ways. Various studies have been carried out to investigate the effects of stimulus significance so manipulated, and these will be briefly reviewed for a number of psychophysiological indices in turn.

Germana (1968) varied stimulus significance by requiring different responses to different stimuli, and found that greater galvanic skin responses (GSRs) were elicited under signal conditions. Korn and Moyer (1968) found that "pay attention" instructions resulted in larger GSRs to the first tone of a series but obtained no evidence of differential habituation rates. Harding and Punzo (1971) carried out a series of experiments which required different types of responses ("pay attention"' vs. skeletal responses) under different degrees of response uncertainty. There was no attentional effect on the magnitude or habituation rate of elicited GSRs, which is surprising in the context of the previous studies. The requirements of a skeletal response of different uncertainty resulted in OR magnitude enhancement 
and reduced habituation rates directly related to the amount of uncertainty. These effects were attributed to the differential habituation rates rather than to differences in the magnitude of the initial OR itself. This study has thus indicated the existence of different effects of attentional and response requirements, contrary to Sokolov's concept of a single dimension of "significance."

With respiratory pause as the OR indicator, only one study by Porges and Raskin (1969) has attempted a systematic variation in stimulus significance levels. These experimenters obtained no effect attributable to this manipulation, but their response measures failed to consider short-latency effects so that the importance of their result is minimal.

The heart rate (HR) indicator of the OR has been investigated to a far greater extent than those considered above, and the work of Graham and her colleagues [developed from the review of the field by Graham and Clifton (1966)] has resulted in the identification of $\mathrm{HR}$ deceleration as the OR indicator to simple nonsignal stimuli. However, with signal stimuli, evidence appears to be mounting in support of different effects of attentional and response manipulation similar to those found with the GSR. For example, Klorman and Lang (1972) found deceleration to be enhanced with signal vs. nonsignal tones when the signal stimuli were warning tones for a simple reaction time (RT) task. In contrast, the imperative RT signal appeared to produce $H R$ acceleration, but was not discussed in detail in their report. The results of Johnson and May (1969) appear to confirm this association of HR acceleration with skeletal response requirements. Subjects in their RT group were required to press a switch when a warning signal occurred and to release it as soon as possible following the imperative signal, while subjects in a time-estimation group were also required to press a switch upon the occurrence of the warning signal but were required to release it an estimated $4 \mathrm{sec}$ later. Thus, in both groups, two motor responses were required. The HR response in all four cases was one of acceleration, with the RT group producing the larger response magnitude. Again, such a separation within the proposed dimension of significance poses problems for Sokolov's theory.

The effect of stimulus significance on peripheral and cephalic pulse volume (PPV and CPV, respectively) has not been extensively investigated. Cohen and Johnson (1971) found a vasoconstrictive PPV response to tonal stimuli which habituated less under signal conditions than under nonsignal conditions. This supports Sokolov's predictions with PPV, but different results were obtained with the CPV which led the authors to conclude that cephalic vasomotor responses failed to behave as predicted by Sokolov. Apart from this study, there is little work of relevance to the problem of significance in these response systems.

With EEG alpha desynchronization as the OR indicator, Holloway and Parsons (1971) found habituation with nonsignal stimuli, while Orr and Stern (1971) found that RT tasks were associated with nonhabituating EEG responses. Gale, Haslum, and Penfold (1971) studied the EEG correlates of cumulative expectancy in vigilance-type tasks and found that alpha activity was reduced significantly in those conditions which required most attention. These three examples illustrate the present state of knowledge with the EEG. Attentional demands appear to result in enhanced responding, but the effect of this signal variable upon habituation rates is unknown. On the other hand, the effect of requiring a motor response such as in a simple RT task appears to be to inhibit habituation of the EEG response, while the effect upon the magnitude of desynchronization has not been established. One possible reason for this paucity of recent data in the area is the upsurge in the use of averaged evoked potentials rather than discrete EEG events.

These results indicate some of the problems existing in this area when "significance" is considered as a determinant of the OR. Overall, there is a major lack of data relevant to the influence of change in this variable upon stimulus-intensity effects and response habituation. Barry $(1975,1976,1977)$ has discussed the effects of intensity and novelty under nonsignal conditions upon the six psychophysiological indices surveyed above. The present study investigated the effects of attentional demand upon these stimulus-response relationships as part of an effort to elucidate the apparently different effects due to attentional and response requirements reported in the literature. These were conceptualized by Sokolov as being similar in nature, resulting in enhanced response magnitude and reduced habituation rates when compared with ORs elicited by nonsignal stimuli. (Further work on the active response side of Sokolov's dimension of "significance" is currently in preparation.)

\section{METHOD}

Twenty-four males aged 18 to 25 participated on a voluntary basis to fulfill a requirement of an introductory psychology course at the University of Sydney. Stimuli consisted of 2-sec, $1,000-\mathrm{Hz}$ tones presented binaurally via circumaural earphones with an interstimulus interval randomly varying between 20 and $100 \mathrm{sec}$ with mean $60 \mathrm{sec}$. Each subject received a unique cycle of the stimulus intensities $20,30,40$, and $50 \mathrm{~dB}$ SPL (one of the 4 ! possible orders of four intensities), effectively confounding individual differences with stimulus order. Calibration was carried out using an artificial ear coupler with a flat-plate adaptor manufactured at the University of Sydney. Eight cycles of the four stimuli were presented to enable habituation to be studied.

The GSR was recorded from lead electrodes on the volar surfaces of the first and second fingers of the subject's left hand 
and written out as ac-coupled resistance changes on an E \& $M$ Physiograph Six with a system time-constant of $5 \mathrm{sec}$ and paper speed of $2.5 \mathrm{~mm} / \mathrm{sec}$. Respiration was also recorded on the Physiograph from an $E$ \& $M$ bellows pneumograph attached around the subject's chest. The EKG was recorded from a standard electrode placement (EKG lead 1) on a Grass IIID polygraph with standard EKG time constant and paper speed of $15 \mathrm{~mm} / \mathrm{sec}$. PPV was recorded from an E \& $M$ photoelectric pulse pickup attached to the distal volar surface of the third finger of the subject's left hand (which rested on the arm of the subject chair) and written out on the Physiograph. Another pulse pickup was placed against the zygoma, where a pulse could be manually felt. It was held in position by the earphones, and its output was recorded as the CPV on the Physiograph. The electroculargram (EOG) was recorded on the Grass polygraph and used to check that the subject's eyes were open as instructed; it will not be discussed further. The subjects were fitted with silver/ silver chloride pad electrodes at $\mathrm{O}_{1}, \mathrm{P}_{3}$, and $\mathrm{C}_{\mathrm{z}}$ (Jasper, 1958). $C_{z}$ was earthed and the activity between $O_{1}$ and $P_{3}$ recorded on the Grass polygraph as the occipital EEG. Stimulus events were simultaneously indicated on a separate channel of each recording machine through the stimulus-presentation system. Subjects sat in the dark with their eyes open, in a comfortable armchair in a sound-reduced light-tight electrically shielded room. They were asked to remain still but awake; the eyes-open condition was designed to assist this last request.

The subjects were told they would receive a series of tones and would be questioned regarding the nature of the tone series at the end of the experiment. Each subject was warned that his results would not be used unless the experimenter was convinced by this postexperimental enquiry of the subject's attention to the tones throughout the series. Although a postexperimental enquiry was carried out to maintain the impact of this attentional demand, the results were not used to exclude any subjects from the group. Thus this manipulation was aimed at modifying the stimulus complex presented to an unselected sample of subjects. This attentional demand distinguished the procedure used here from that used to obtain the information previously reported in Barry $(1975,1976,1977)$ regarding the OR produced by nonsignal stimuli, where the stimuli were implied to be irrelevant elevator noises. (The headphones were supposedly used to help mask such noises.) Apart from the subject sample and the attentional demand, the present procedure replicated that previously reported. The experimental analysis reported here is thus based on a threeway design (over attention, stimulus intensity, and novelty), with repeated measures on the last two variables.

\section{RESULTS}

The results of the experiment are presented below for each physiological indicator in turn.

\section{GSR}

The GSR was defined as a measurable drop in resistance with latency between 1 and $5 \mathrm{sec}$ from stimulus onset. The mean values of GSR magnitude are displayed as a function of intensity in Figure 1 and as a function of repeated stimulus cycles in Figure 2. The distribution of scores was far from normal (due to the large number of zero scores), precluding the use of parametric data analysis. A Friedman analysis of variance by ranks (Siegel, 1966) carried out on the scores averaged over trials showed the intensity effect to be significant well beyond the .05 level $\left(\chi_{\mathrm{r}}^{2}=20.5\right.$, df $\left.=3\right)$ as was the habituation effect exhibited by the scores averaged over
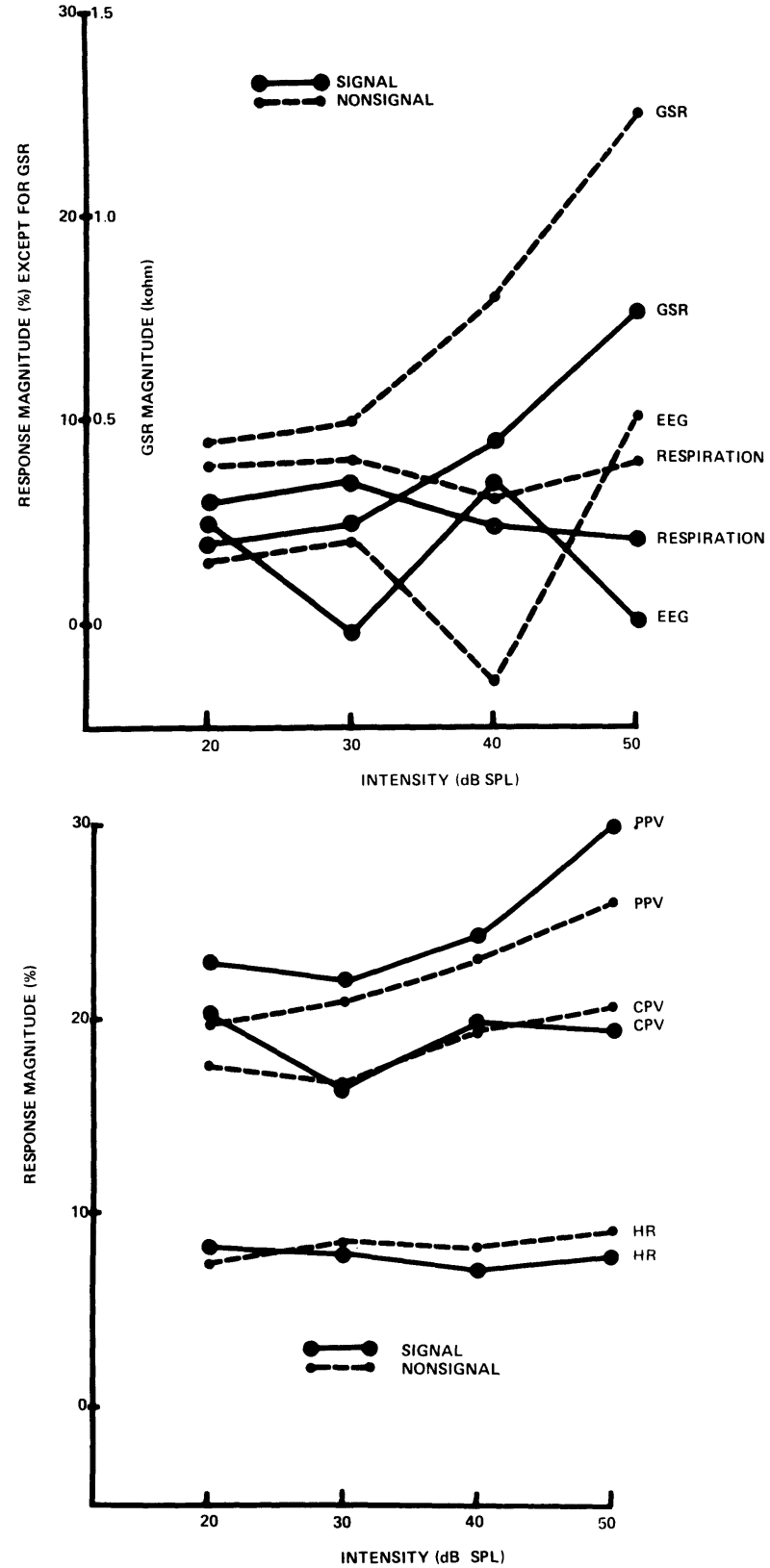

Figure 1. Response magnitude as a function of stimulus intensity. Top graph shows those measures which habituated; nonhabituating measures are shown on the bottom.

intensities $\left(\chi_{\mathrm{r}}^{2}=38.2\right.$, df $\left.=7\right)$. A comparison of these results with those reported in Barry (1975) indicated that attentional demand produced a consistently smaller average response at all data points. This was confirmed by a significant Mann-Whitney U test (Siegel, 1966) comparing the average response over all stimulus presentations between individuals in the two experiments $\left(\mathrm{U}=374.5, \mathrm{~N}_{1}=\mathrm{N}_{2}=24\right)$. A comparison of response frequencies obtained in the two experiments confirmed this reduced level of responding. 

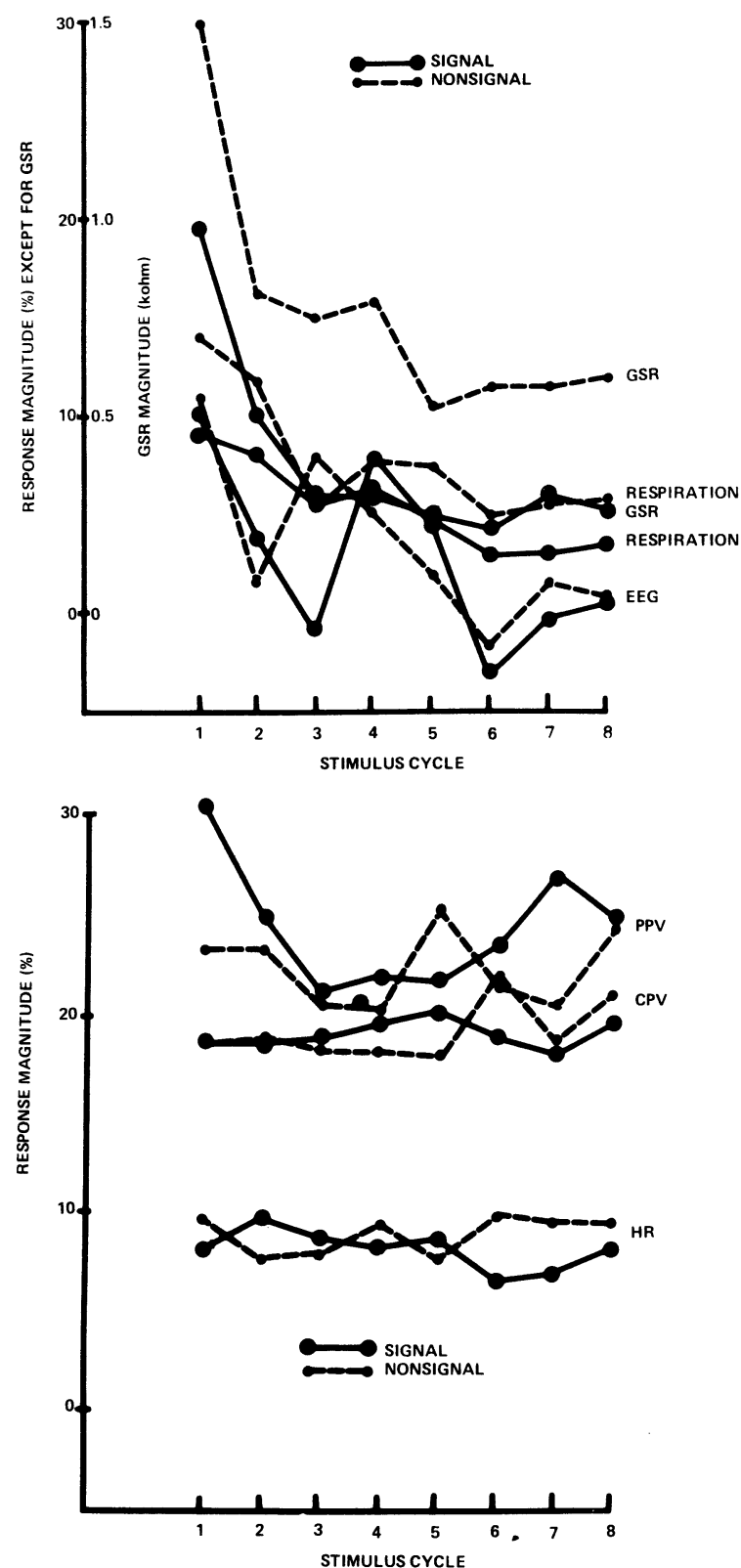

Figure 2. Response magnitude as a function of repeated stimulus cycles. Top graph show's habituating measures; nonhabituating measures are shown on the bottom.

\section{Respiration}

For each stimulus event, the periods of the respiratory cycle (measured from inspiration onset) immediately before stimulation and the cycle containing the stimulus onset were obtained. The percentage increase in respiratory period was calculated for each stimulus presentation. Mean values are displayed against stimulus intensity in Figure 1 and against stimulus cycles in Figure 2. The data from Barry (1977) and the present experiment were subjected to a three-way analysis of variance over the factors of attention, intensity, and repetition, with repeated measures trend analyses on the last two variables. Even though there was an apparently consistent reduction in response magnitude associated with attentional demand, this failed to reach significance $[\mathrm{F}(1,46)=2.50, \mathrm{MSe}=557.21]$. There was no significant intensity effect, but the strong habituation effect was confirmed, mainly appearing as a linear trend over trials $[\mathrm{F}(1,322)=41.20$, MSe $=221.32]$ and with some quadratic component $[F(1,322)=4.40]$. There were no significant interactions. Thus, apart from a nonsignificant reduction in response magnitude, the manipulation of significance level in this study has had no effect upon the respiratory index of the $O R$.

\section{HR}

The form of the HR response was investigated as a preliminary step in the analysis of the HR index of the OR and found to consist of a simple fleeting deceleration "peaking" at about Poststimulus Beat 3. This was virtually identical to that reported in Barry (1977) and the same criteria were used here in extracting response magnitudes from subject protocols. The HR response was defined as the maximum deceleration relative to prestimulus $H R$ occurring in the first five poststimulus beats. This was converted to a percentage decrease in HR from Prestimulus Beat 1 , and mean values of this response as a function of intensity and novelty are shown in Figures 1 and 2. These data and those of the previous experiment were subjected to a three-way ANOVA as used for respiration. This indicated no significant main effects of intensity, novelty, or significance, and no significant interactions. The results of this experiment confirm the findings reported in Barry (1977) regarding intensity and novelty effects on the HR response and indicate that attentional demand has had no effect upon this index of the OR.

\section{PPV}

The smallest pulse amplitude occuring between 7 and 12 beats poststimulus was taken as the point of maximum response, as in the previous experiment. This response was converted to a percentage decrease in PPV by comparison with the amplitude of Prestimulus Beat 1 and averaged to obtain the values plotted in Figures 1 and 2. The raw percentages were analyzed as before, indicating significant intensity and repetition effects, but no significant effect of enhanced attentional requirements. The intensity effect was primarily described by a linear trend $[\mathrm{F}(1,138)=32.21, \mathrm{MSe}=388.44]$ and a smaller quadratic trend $[F(1,138)=5.90]$. There was a significant stimulus repetition effect due to a quadratic trend $[\mathrm{F}(1,322)=8.92, \mathrm{MSe}=346.23]$ which could not be conceptualized as indicating simple habituation with decreasing stimulus novelty. Although 
not reaching statistical significance, there was an increase in response magnitudes of approximately $10 \%$ compared with results in the previous nonsignal investigation. There were no significant interactions.

\section{CPV}

The form of the CPV response was investigated as in the previous experiment (Barry, 1977), and a similar dilation, peaking approximately at Poststimulus Beat 3, was obtained. The magnitude of this response was extracted from the protocols using the latency criteria defined in the previous experiment ( 1 to 6 beats poststimulus) and converted to a percentage change from Prestimulus Beat 1 . These values were subjected to the three-way ANOVA of the previous indices, which confirmed the lack of sensitivity apparent from Figures 1 and 2: there were no significant main effects or interactions, confirming the results of the previous study and indicating no significant effect attributable to attentional demand.

\section{EEG}

The amplitude of occipital alpha activity was hand-scored for the 1-sec intervals before and after each stimulus onset, as described in Barry (1976) and Barry and Beh $(1972,1976)$. The percentage change at stimulus onset is displayed in Figures 1 and 2. The raw values of this response were analyzed as above. This analysis indicated a linear trend over stimulus cycles $[\mathrm{F}(1,322)=10.05, \mathrm{MSe}=1,119.84]$ but no other significant main effect. (Overall, the mean level of responding is some $18 \%$ below that obtained in the previous experiment, but, as occurred with the respiratory index of the $O R$, this failed to reach significance.) There was a significant interaction between the cubic trends over intensity obtained in the two experiments $[\mathrm{F}(1,138)=13.50, \mathrm{MSe}=$ 1084.88], and this provides some conceptual difficulties which will be discussed later. There were no other significant interactions.

\section{DISCUSSION}

Sokolov (1963) predicted that attentional demand should enhance the significance attributed by subjects to the stimulus array. Such enhanced significance was claimed to produce enhanced response magnitudes and a reduced habituation rate. With the design of analysis employed here, these should have been detectable as a simple effect due to significance and an interaction between stimulus repetition effects and significance, respectively. Such effects were not found with any of the six psychophysiological indices recorded in this study. Rather, this experiment has done much to confirm the validity of the findings of the previous experiment with regard to the consistency of the stimulus-response relationships obtained there rather than finding marked changes in these relationships as significance was varied. In essence, these relationships appear markedly robust, particularly when it is considered that the two experiments were performed with independent groups of subjects who were not screened in any way which would restrict the range of physiological responses sampled.

The GSR results indicated a reduction in response magnitude associated with an apparent increase in stimulus significance. Similar, but nonsignificant, reductions in response magnitude were found with respiration and EEG alpha desynchronization. These effects are contrary to Sokolov's predictions and will be discussed further. PPV dilation was enhanced in this experiment as predicted by Sokolov, but this effect failed to reach statistical significance. No such effects were apparent with HR or CPV indices of the $O R$, and in no case was there an interaction between habituation rate and significance level. This failure to obtain the predicted effects of stimulus significance must be viewed as embarassing for Sokolov's position.

When examining these results for some regularizing principle, it becomes apparent that the three indices that exhibit reduced magnitude in the present experiment (GSR, respiration, and EEG) are also the only indices which exhibit habituation with stimulus repetition. This generates the hypothesis that both these effects are associated with the sensitivity of these indices to the novelty of the stimulus rather than significance per se. Reflection upon the procedure undertaken to enhance attention in this experiment indicates that this may also have resulted in decreased novelty of the stimulus complex: subjects were told that they were to receive a series of tones, whereas in the previous experiment the tones were not mentioned. Thus the two experiments differ in both attentional demand and stimulus novelty, and, while the importance of this was not previously apparent, it now allows extended hypotheses regarding the systematic labeling of the response systems which appear to be emerging in place of Sokolov's unitary OR.

The GSR, respiration, and EEG may thus be conceptualized as being sensitive to stimulus novelty and not attentional demand, while the PPV response may be sensitive to attentional requirements bui not novelty. Of these indices, the GSR and PPV are sensitive to variation in stimulus intensity, while respiration is not. EEG alpha desynchronization was found not to be systematically related to stimulus intensity overall, but showed conflicting cubic trends over intensity in the two experiments. Such complexity is of doubtful psychological significance and may be taken, with due caution, to indicate the absence of a reliable stimulus-intensity effect with this index. The remaining two indices, $\mathrm{HR}$ and CPV, have been found to be insensitive to variation in significance, intensity, and novelty. 
This analysis suggests that the response measures considered here reflect the activity of at least three response systems involved in the perceptual process rather than a simple unitary system as proposed by Sokolov. For ease of conceptualization, these may be labeled in the following manner, but it must be stressed that the labels used represent only summary statements rather than explanatory or functional devices:

\section{Stimulus register: These responses occur at every stimulus presentation regard- less of intensity or novelty: $H R$ deceleration and $C P V$ dilation.}

Intensity register: These responses reflect differences in stimulus intensity: GSR and PPV constriction.

Novelty register: These responses reflect differences in the novelty of the stimulus complex: $G S R$, respiration pause and EEG desynchronization.

It is apparent that some physiological responses are influenced by more than one system, but such complexity need not be surprising: if the human organism responded at a simple physiological level in the same manner, with all responses occurring together as proposed by Sokolov, then psychophysiology could be expected to be a closed body of knowledge rather than in its present state of flux. In fact, this complexity does much to allow the diversity of results found in the literature and provides a more elaborate structure to encompass the vast array of previously contradictory findings.

In summary, comparison of the results of these two experiments has enabled the elaboration of a new conceptual system in place of the unitary OR concept. This new conceptualization posits three systems involved in the perceptual process and sensitive to the occurrence of the stimulus per se, to stimulus novelty, and to intensity. Each of these systems is hypothesized to determine (either completely or partly in the case of different measures) the response magnitude of two or more of the physiological responses studied here. It is also to be noted that attention has failed to emerge as a valid dimension affecting OR magnitude in that all effects associated with the attentional manipulation (with the possible exception of the PPV response) are able to be explained satisfactorily by reference to novelty: considerations of theoretical parsimony indicate that the concept of attention needs refinement beyond the casual level of usage employed here and in previous OR studies.
Overall, these results add to the growing list of reports which cannot be encompassed within Sokolov's simplistic unitary OR concept. The tentative conceptualization offered here should be useful in generating a new appraisal of the problems existing in this area of psychology.

\section{REFERENCES}

BARRY, R. J. Low-intensity auditory stimulation and the GSR orienting response. Physiological Psychology, 1975, 3, 98-100.

BARRY, R. J. Failure to find the "local" EEG OR to low-level auditory stimulation. Physiological Psychology, 1976. 4. 171-174.

BARRY, R. J. Failure to find evidence of the unitary OR concept with indifferent low-intensity auditory stimuli. Physiological Psychology, 1977, 5, 89-96.

BARRY, R. J., \& BEH, H. C. EEG desynchronization as a function of intensity of visual stimulation. Psychonomic Science. 1972, 26, 241-242.

BARRY, R. J., \& BEH. H. C. EEG correlates of the afterimage of visual stimulation. Psychophysiology, 1976, 13, 75-80.

Cohen, M. J.. \& Johnson, H. J. Effects of intensity and the signal value of stimuli on the orienting and defensive responses. Journal of Experimental Psychology, 1971, 88, 286-288.

Gale, A., Haslum, M.. \& Penfold, V. EEG correlates of cumulative expectancy and subjective estimates of alertness in a vigilance-type task. Quarterly Journal of Experimental Psychology, 1971, 23. 245-254.

Germana, J. Response characteristics and the OR. Journal of Experimental Psychology, 1968, 78, 610-616.

GrahaM, F. K. Habituation and dishabituation of responses innervated by the autonomic nervous system. In $H$. V. $S$. Peeke \& M. J. Herz (Eds.), Habituation. New York: Academic Press, 1973.

Graham, F. K., \& Clifton, R. K. Heart-rate change as a component of the orienting response. Psychological Bulletin, 1966. 65, 305-320.

Harding, G., \& Punzo, F. Response uncertainty and skin conductance. Journal of Experimental Psychology. 1971. 88. 265-272.

Holloway, F. A., \& Parsons, O. A. Habituation of the orienting reflex in brain damaged patients. Psychophysiology. $1971,8.623-634$.

JASPER, H. H. Report of the committee on methods of clinical examination in electroencephalography. Electroencephalography and Clinical Neurophysiology, 1958, 10, 370-375.

Johnson, H. J.. \& MAY, J. R. Phasic HR changes in RT and time estimation. Psychophysiology, 1969, 6. 351-357.

Klorman, R., \& LANG, P. J. Cardiac responses to signal and nonsignal tasks in 9-year-olds. Psychonomic Science, 1972, 28. 299-300.

Korn, J. H., \& Moyer, K. E. Effects of set and sex on the electrodermal orienting response. Psychophysiology, 1968, 4. 453-459.

ORR, W. C., \& STERN, J. A. The relationship between stimulus information and reaction time, and cortical habituation. Psychophysiology, 1971, 7, 475-484.

Porges, S. W., \& Raskin, D. C. Respiratory and heart rate components of attention. Journal of Experimental Psychology, 1969, 81, 497-503.

SIEgEL, S. Nonparametric statistics for the behavioral sciences. New York: McGraw-Hill, 1966.

Sokolov, E. N. Perception and the conditioned reflex. Oxford: Pergamon Press, 1963.

(Received for publication August 12, 1976; revision accepted January $14,1977$. 